\title{
Neural Monitoring in Thyroid Surgery: Is It Evidence Based? Is It Cost Effective?: Reply
}

\author{
Jennifer L. Marti ${ }^{1}$ - Gregory Randolph ${ }^{2}$
}

Published online: 8 June 2016

(C) Société Internationale de Chirurgie 2016

\section{Dear Editor,}

We appreciate the commentary of Dr. Anuwong and colleagues. We certainly agree that value and cost effectiveness considerations cannot always be seamlessly applied to emerging technologies. Our data indicate that intraoperative nerve monitoring (IONM) in thyroid surgery is clearly viewed by the majority of specialist surgeons as providing value. Nevertheless, surgeons must always be conscientious about new technologies in the operating room. We should ensure that access to IONM is provided in a manner that does not result in unacceptably increased costs to patients or the health care system. Surgical and technologic advances may be associated with costs, which fellowshiptrained thyroid surgeons judge acceptable in the added value they bring.

Sincerely,

Jennifer L Marti MD FACS,

Gregory Randolph MD FACS FACE

Jennifer L. Marti

drjennifermarti@gmail.com

1 NewYork-Presbyterian/Weill Cornell Medical Center, New York, USA

2 Massachusetts Eye and Ear Infirmary, Boston, USA 